ISSN 1991-8631

Original Paper

http://indexmedicus.afro.who.int

\title{
Etude de quelques récoltes de Crepidotus (Fr.) Quélet sur Eucalyptus sp. (Nord-Ouest du Maroc)
}

\author{
Ahmed OUABBOU * , Amina OUAZZANI TOUHAMI, Rachid BENKIRANE et \\ Allal DOUIRA
}

Université Ibn Tofaïl, Faculté des Sciences, Laboratoire de Botanique et de Protection des Plantes, B.P. 133, Kenitra, Maroc.

*Auteur correspondant, E-mail: douiraallal@hotmail.com

\section{RESUME}

Dans le but de contribuer à l'étude de la flore fongique du Maroc, des sorties ont été organisées dans la région du Gharb (Nord-Ouest du Maroc) pour récolter les espèces du genre Crepidotuqs, champignons Basidiomycètes lignicoles. Les caractères macroscopiques et microscopiques des spécimens récoltés sont étudiés respectivement sur terrain et sur coupes minces de l'épicutus, des lames et de stipe. Crepidotus mollis var. squamulosus et Crepidotus calolepis (Fries) Karsten identifiées dans cette étude sont comparés avec Crepidotus calolepis var. squamulosus (Cout.) Senn-Irlet (1995) et Crepidotus calolepis mollis var. calolepis (Fr.) P. Karst. (1879) décrites par Senn-Irlet (1995). Ces comparaisons ont été discutées également selon les observations de Malençon et Bertauld (1975) effectuées sur le genre Crepidotus au Maroc. De ces résultats, Crepidotus calolepis (Fries) Karsten peut être considérée comme nouvelle espèce pour le Maroc.

(C) 2012 International Formulae Group. All rights reserved.

Mots clés: Maroc, Gharb, Basidiomycètes, Crepidotus mollis var.squamulosus, Crepidotus calolepis, description.

\section{INTRODUCTION}

Chez les Macromycètes, on trouve deux phylums: Ascomycota et Basidiomycota, qui diffèrent essentiellement par la présence des asques chez les premiers et de basides chez les seconds. Chez ces champignons supérieurs, la systématique fait appel à tous les caractères, aussi bien ceux tirés de l'aspect extérieur que ceux qui résultent du développement et de l'anatomie du carpophore (Maublanc, 1926); ainsi, l'identification d'un champignon implique tous les organes des sens et une fine observation des différents critères (Loiseau 1951) :
- forme et taille des différents éléments du carpophore; habitat du champignon et saison d'apparition des carpophores; caractéristiques du chapeau, de la chair, et du stipe; forme de l'hyménium; odeur et saveur; réactions macrochimiques des différentes parties du carpophore; couleur et caractéristiques de la partie fertile; couleur des spores.

Les espèces du genre Crepidotus sont de petite taille, lignicoles, d'aspect pleurotoïde, à chair très molle (Bodeny, 1966). Le pied est court, excentrique ou même complètement latéral; certaines espèces sont dépourvues de pied. Les spores sont ochracées. 
$\mathrm{Au}$ Maroc, Malençon et Bertault (1975) ont signalé la présence de Crepidotus mollis (Schaeff.: Fr.) Staude et d'une variété squameuse dans quelques régions du Maroc (Rabat, Tanger, Rif, El Jadida), sans toutefois les décrire. Ces deux auteurs n'ont pas utilisé l'épithète Crepidotus calolepis (Fries) Karsten pour cette variété et ont conservé l'appellation $C$. mollis var. squamulosis $\mathrm{P}$. Coutinho.

Dans cette étude, deux espèces du genre Crepidotus (Fr.) Quélet ont été rencontrées dans la région du Gharb sur Eucalyptus: Crepidotus calolepis (Fries) Karsten et Crepidotus mollis var squamulosus $\mathrm{P}$. Coutinho. Ces deux espèces sont décrites et illustrées puis comparées avec deux variétés décrites par Senn-Irlet: Crepidotus calolepis var. squamulosus (Cout.) Senn-Irlet (1995), et Crepidotus calolepis var. calolepis (Fr.) P. Karst. (1879). La monographie de Consilgio et Ledo (2008) a été consultée pour confirmer la détermination des récoltes.

\section{MATERIEL ET METHODES}

Les spécimens du Crepidotu, récoltés pendant l'hiver et le printemps de 2009 sur les troncs vivants d'Eucalyptus sp. dans la Kasbah de Mehdia et la ville de Kénitra (Figure 1), peuvent exister sur le même tronc. Les exsiccata de ces espèces sont conservés au Laboratoire de Botanique et de Protection des Plantes sous les références $\mathrm{CC} 10$ et CC11.

La récolte des Crepidotus en vue de leur identification est une opération qui mérite beaucoup d'attention. Sur le terrain, on note prudemment certains caractères très évanescents sur le chapeau, la marge, l'arête des lames et le stipe. On mentionne précisément la période de la récolte du carpophore et de son habitat (nature du terrain, associations végétales environnantes, etc.).

Les échantillons sont étudiés en laboratoire, en particulier les caractères suivants :

- caractères morphologiques: aspect général à l'état jeune et à l'état adulte du chapeau du pied, des lames, de la chair et autres traits remarquables avant que le champignon ne se détériore ;

- caractères organoleptiques: couleur (cuticule, lames, pied, chair, sporée), saveur, odeur, viscosité, aspect de la cuticule ;

- caractères microscopiques observés sur des coupes, des écrasements ou à partir des spores récoltées; les dimensions des spores, des cystides, des basides et des stérigmates ont été confrontées avec les données disponibles dans la littérature.

Les descriptions macroscopiques et microscopiques des spécimens récoltés sont comparées avec celles données par Senn-Irlet (1995) (Tableaux 1, 2, 3 et 4).

L'identification des espèces est faite après consultation de quelques clés de détermination: Senn-Irlet (1995), Romagnesi (1995), Courtecuisse et Duhem (2000), Roux (2006), Consilgio et Ledo (2008), Courtecuisse (2009), Peric et Peric (2010).

\section{RESULTATS}

Deux espèces ont été décrites dans cette étude:

Crepidotus calolepis (Fr.) P. Karst. 1879. Récolte de 2009 sur Eucalyptus gomphocephala, Gharb (Nord-Ouest Maroc).

Le chapeau (15 à $25 \mathrm{~mm}$ de diamètre) est semi-circulaire, réniforme, convexe, à marge infléchie, villeux au point d'attache, hygrophane, méchuleux et de couleur jaune clair. Les méchules sont brunâtres (Figure 2A). Le stipe est visible chez les jeunes fructifications. Les lames sont assez serrées, arquées, adnées et de couleur blanchâtre puis jaune clair. Elles mesurent $11 \mathrm{~mm}$ de longueur et $3 \mathrm{~mm}$ de largeur (Figure 2B). Les spores (7 à $8 \times 4$ à $5 \mu \mathrm{m}, 1,3<\mathrm{Q}<1,7)$ sont lisses, à paroi épaisse, ellipsoïdales amygdaliformes et de couleur rosâtre (Figure $5 \mathrm{~J}$ et $\mathrm{K}$ ). Les basides $(20 \times 6,6 \mu \mathrm{m}$. $)$ sont tétrasporiques (Figure $4 \mathrm{I}$ et L). Les cheilocystides $(26,5 \times 7$ $\mu \mathrm{m})$ sont cylindriques, utriformes, hyalines et lagéniformes (Figure 4F et G). Les hyphes des écailles mesurent de 7 à $8 \mu \mathrm{m}$ de largeur. La couche gélatineuse est absente au niveau de revêtement (Figure 3E). 


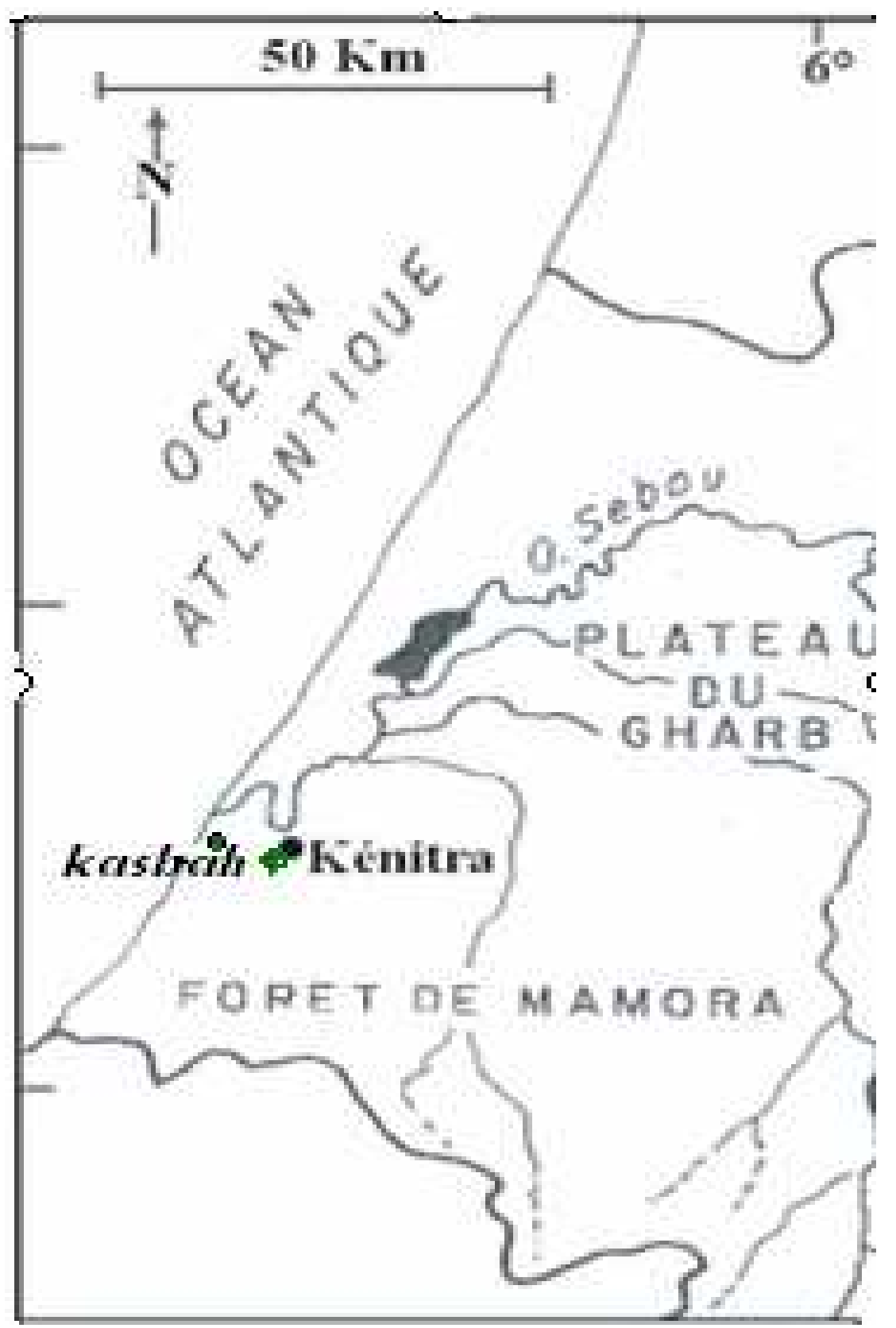

Figure 1: Situation géographique (Bouab, 2008) et localisation des zones de récolte. 
Crepidotus mollis var squamulosus Cout. Récolte de 2009 sur Eucalyptus gomphocephala, Gharb (Nord-Ouest Maroc). Le chapeau (40 $\mathrm{mm}$ de diamètre) est arrondie, circulaire, flabelliforme, à marge largement infléchie, villeux au point d'attache et hygrophane. Il présente de méchules rosâtres ou brunâtres (Figure 6A). La couleur du fond est jaune-brun clair. Le stipe est visible chez les jeunes fructifications. Les lames sont assez serrées, arquées, adnées et de couleur brunâtre. Elles mesurent $12 \mathrm{~mm}$ de longueur et $3 \mathrm{~mm}$ de largeur (Figure 6B).
Les spores $(7-10 \times 5-7 \mu \mathrm{m} 1,3<\mathrm{Q}<$ 1,7) sont lisses, à paroi épaisse, ellipsoïdales, inéquilatérales et de couleur rosâtre (Figure $9 \mathrm{H})$. Les basides $(23 \times 8$ $\mu \mathrm{m})$ sont tétrasporiques (Figure 9G). Les cheilocystides $(29-32 \times 7-8 \mu \mathrm{m})$ sont cylindriques, flexueuses et hyalines (Figure 8F). Les hyphes des écailles mesurent de 8 à $10 \mu \mathrm{m}$ de largeur. Le revêtement présente une couche gélatineuse épaisse (Figure 7D).
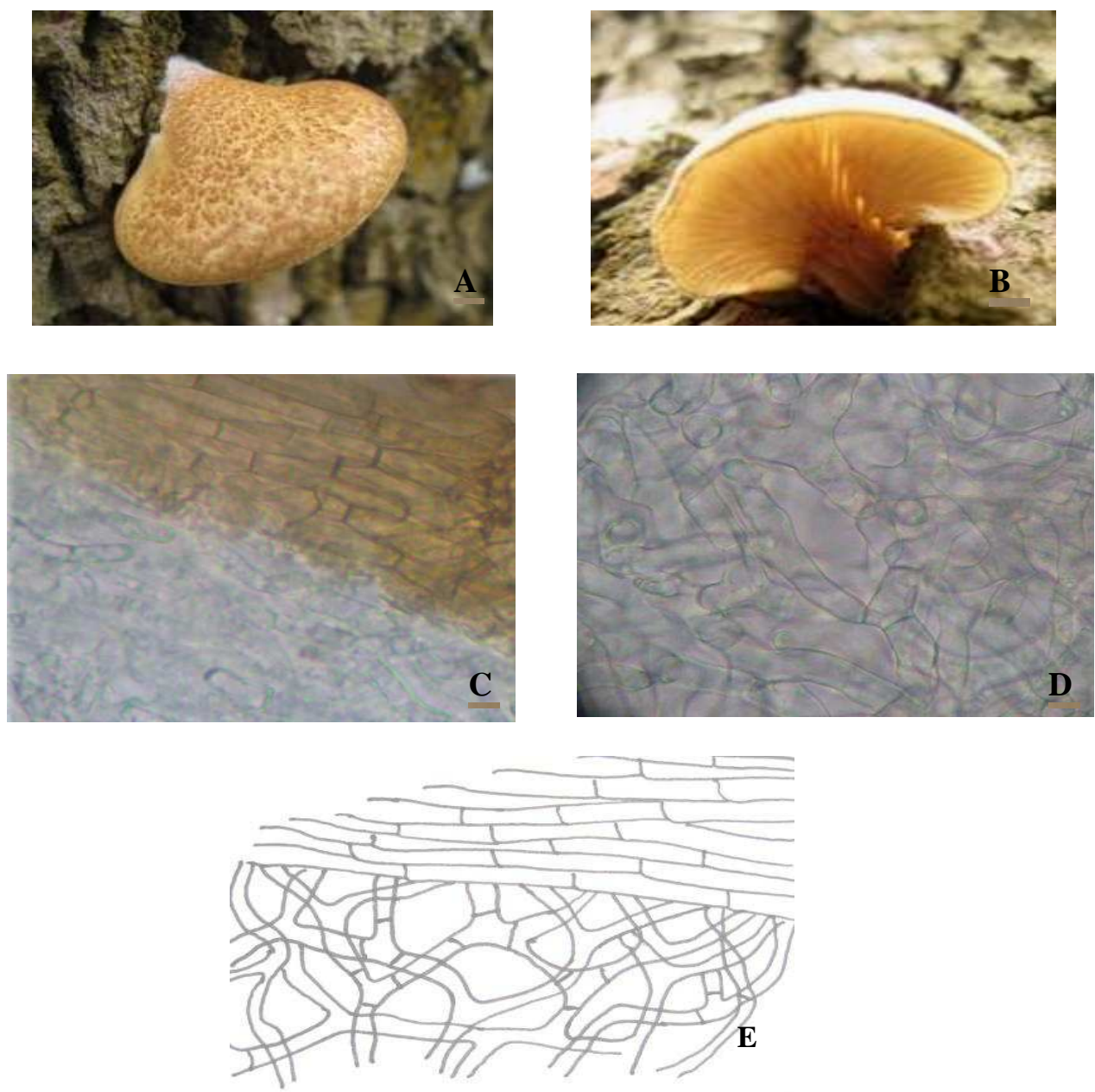

Figure 2: Epicutis de Crepidotus calolepis sous microscope (X 400, montage dans l'eau.): deux couches bien distinctes (C), les hyphes de la surface sont soudés et pigmentés, ceux de la couche inférieure sont lâches (D). (E): schéma. 

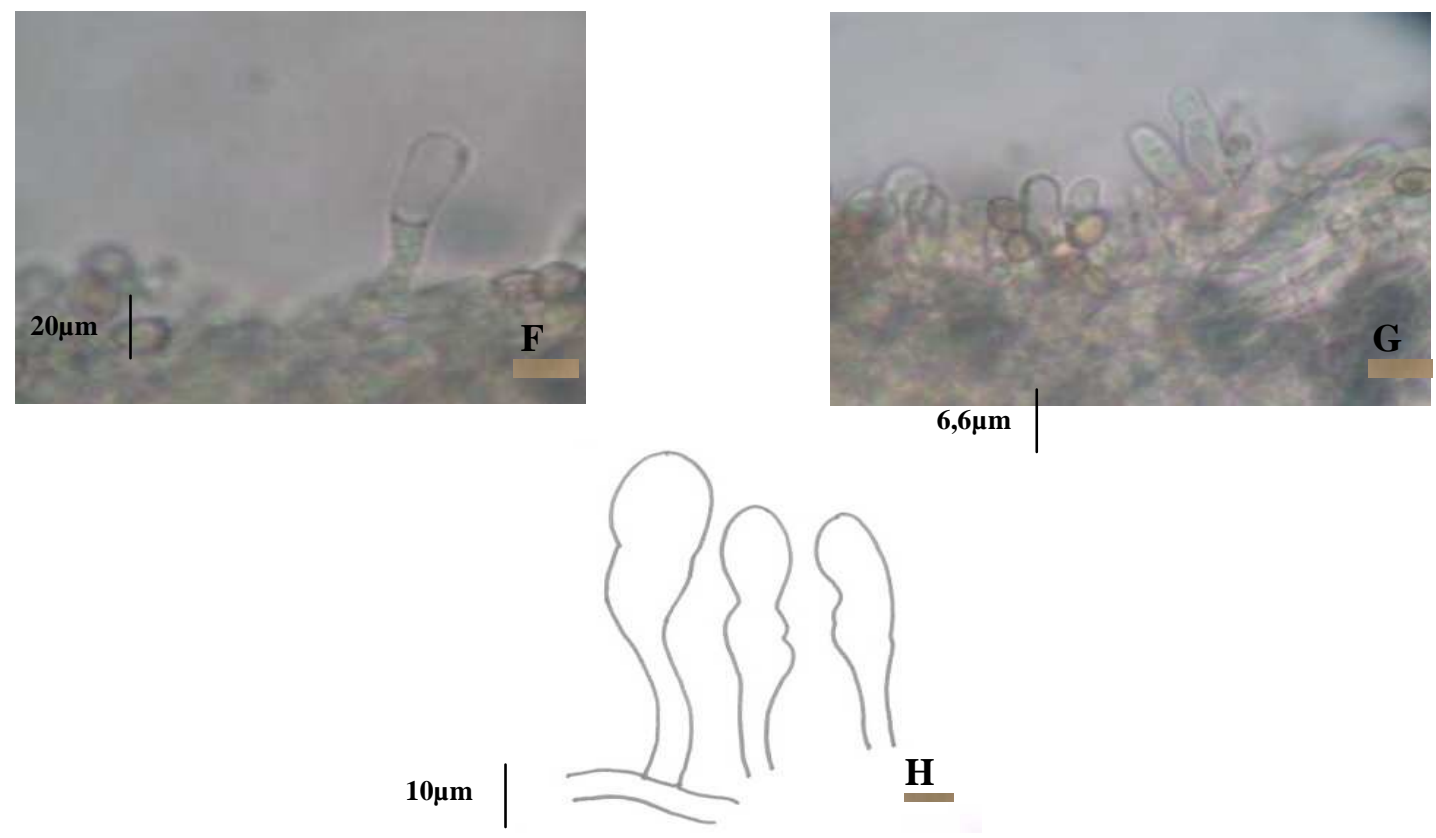

Figure 3: (F) et (G): Types de cheilocystides de Crepidotus calolepis (x 400, montage dans l'eau). (H) : schéma de cheilocyctides.
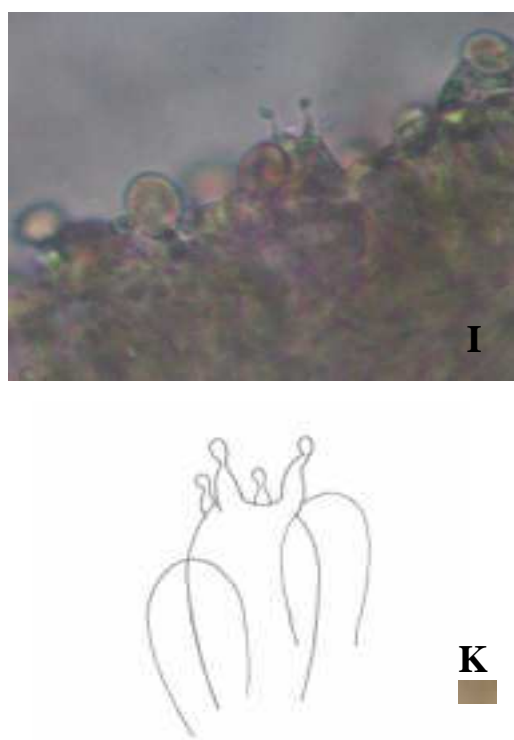

$\mathbf{K}$

$4,4 \mu \mathrm{m}$
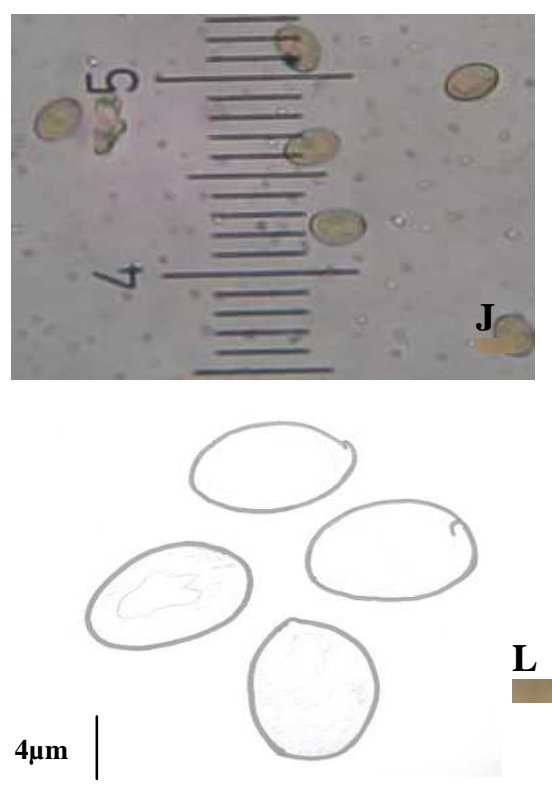

Figure 4: (I et K): Basides et stérigmates (X 400); (I et L): basidiospores (X 400, montage dans l'eau.) de Crepidotus calolepis. 

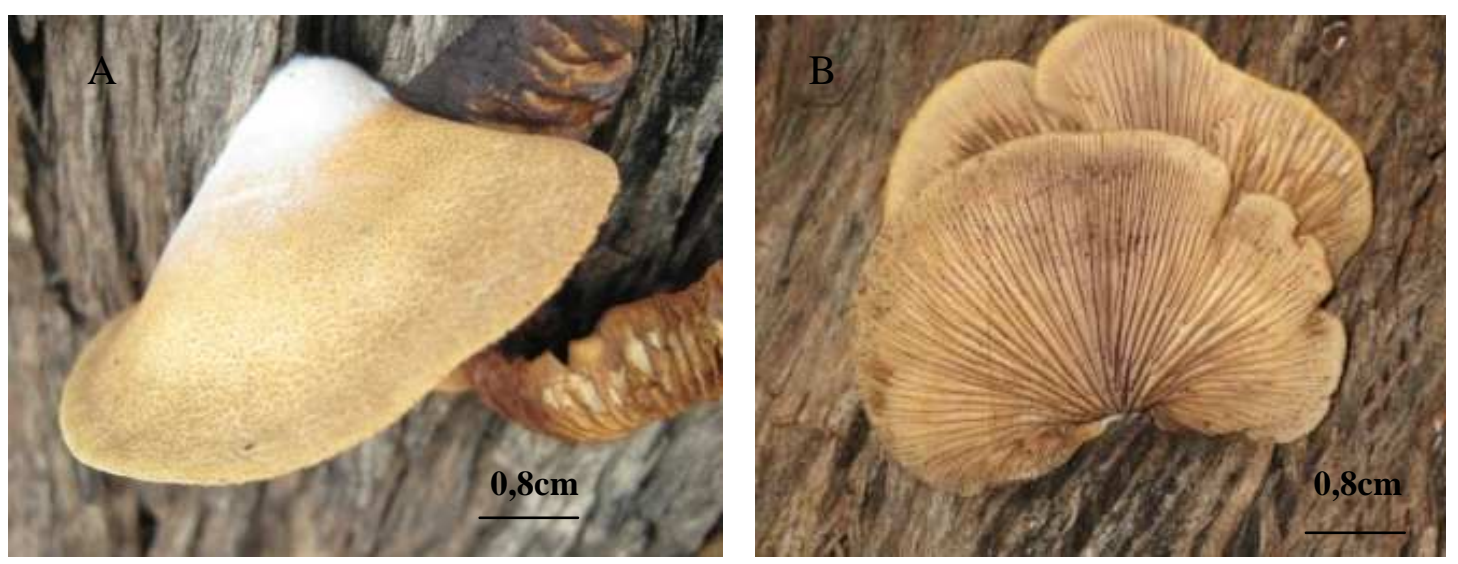

Figure 5: Crepidotus mollis var. squamulosus: Surface du chapeau (A) et insertion des lames (B).

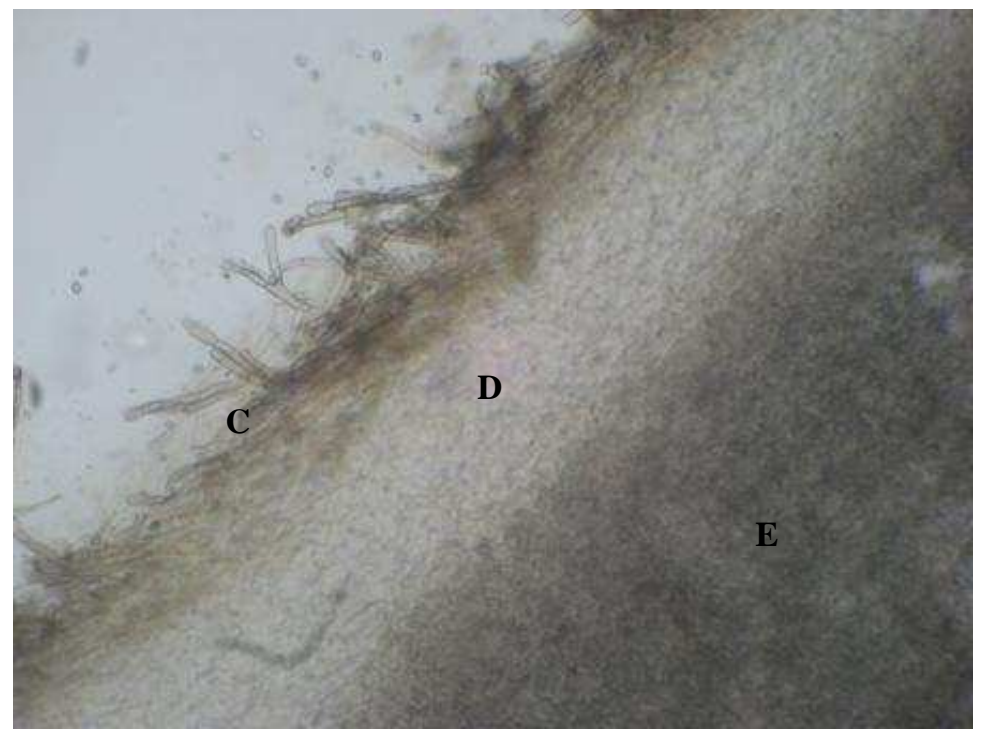

Figure 6: Epicutis de Crepidotus mollis var. squamulosus sous microscope (X 400, montage dans l'eau): trois couches bien distinctes, les hyphes de la surface sont pigmentés (C), la couche gélatineuse (D), les hyphes de la couche inférieure sont lâches (E). 

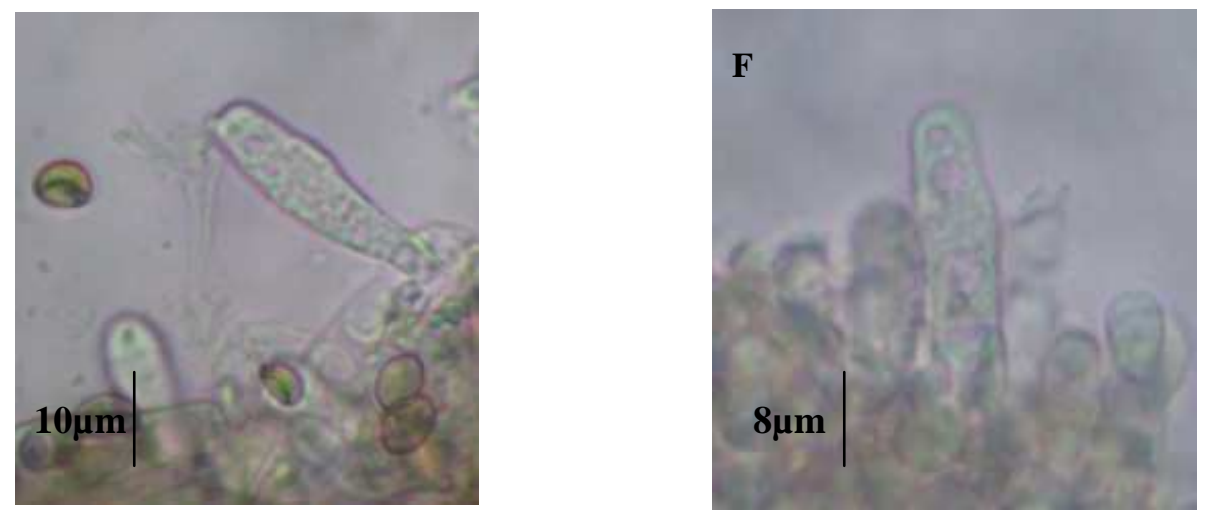

Figure 7: (F) : Types de cheilocystides de Crepidotus mollis var. squamulosus (x 400, montage dans l'eau).
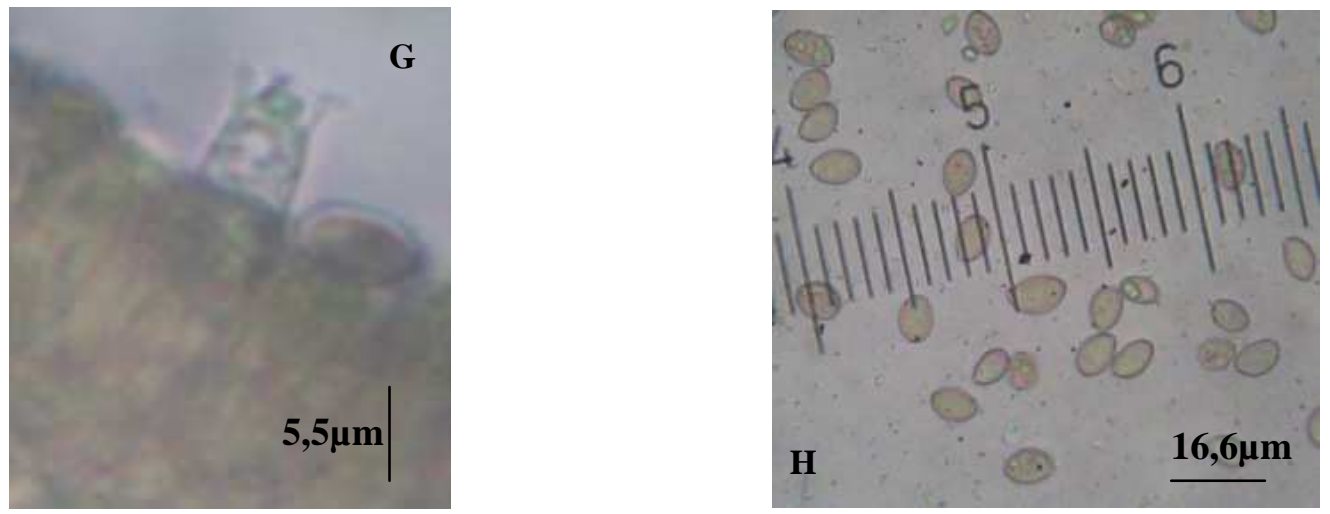

Figure 8: (G): Basides et stérigmates (X 400); (H): basidiospores (X 400, montage dans l'eau) de Crepidotus mollis var. squamulosus. 
Tableau 1: Description macroscopique de Crepidotus calolepis var. calolepis.

\begin{tabular}{|c|c|c|}
\hline $\begin{array}{l}\text { Earactères } \\
\text { microscopiques }\end{array}$ & $\begin{array}{l}\text { Crepidotus calolepis } \\
\text { Récolte de } 2009 \text { sur Eucalyptus } \\
\text { gomphocephala, Gharb (Maroc). }\end{array}$ & $\begin{array}{l}\text { Crepidotus calolepis } \\
\text { var. calolepis. } \\
\text { Senn-Irlet (1995) }\end{array}$ \\
\hline Chapeau & $\begin{array}{l}\text { Taille: } 15-25 \mathrm{~mm} \\
\text { Forme: semi-circulaire, réniforme, } \\
\text { convexe, à marge infléchie, villeux } \\
\text { au point d'attache, hygrophane; } \\
\text { présence de méchules brunâtres } \\
\text { (Figure } 2 \mathrm{~A} \text { ). } \\
\text { Couleur: jaune clair }\end{array}$ & $\begin{array}{l}\text { Taille: } 16-55(10 \mathrm{~mm}) \\
\text { Forme: semi-circulaire, réniforme, } \\
\text { convexe, à marge infléchie, villeux } \\
\text { au point d'attache, hygrophane; } \\
\text { présence d'écailles brunes. } \\
\text { Couleur: ochracé pâle à légèrement } \\
\text { fauve ochracé. }\end{array}$ \\
\hline Stipe & $\begin{array}{l}\text { Visible chez les jeunes } \\
\text { fructifications. }\end{array}$ & $\begin{array}{l}\text { Visible chez les jeunes } \\
\text { fructifications. }\end{array}$ \\
\hline Lames & $\begin{array}{l}\text { Taille: } 11 \times 3 \mathrm{~mm} \\
\text { Forme: assez serrées, arquées, } \\
\text { adnées (Figure } 2 \mathrm{~B}) \text {. } \\
\text { Couleur: blanchâtre puis jaune } \\
\text { clair. }\end{array}$ & $\begin{array}{l}\text { Taille: } 14-18 \times 2-3 \mathrm{~mm} \text {. } \\
\text { Forme: assez serrées, arquées, } \\
\text { adnées. } \\
\text { Couleur: crème puis légèrement } \\
\text { chamois. }\end{array}$ \\
\hline
\end{tabular}

Tableau 2: Description microscopique de Crepidotus calolepis.

\begin{tabular}{|c|c|c|}
\hline $\begin{array}{l}\text { Caractères } \\
\text { microscopiques }\end{array}$ & $\begin{array}{l}\text { Crepidotus calolepis } \\
\text { Récolte de } 2009 \text { sur Eucalyptus } \\
\text { gomphocephala, Gharb(Maroc). }\end{array}$ & $\begin{array}{l}\text { Crepidotus calolepis } \\
\text { var. calolepis. } \\
\text { Senn-Irlet (1995) }\end{array}$ \\
\hline Spores & $\begin{array}{l}\text { Taille: } 7-8 \times 4-5 \mu \mathrm{m} . \\
\text { Lisses, à paroi épaisse, } \\
\text { ellipsoïdales, amygdaliformes, de } \\
\text { couleur rosâtre (Figure } 5 \mathrm{~J} \text { et } \mathrm{K} \text { ). } \\
1,3<\mathrm{Q}<1,7\end{array}$ & $\begin{array}{l}\text { Taille: } 7,5-10 \times 5-7 \mu \mathrm{m} . \\
\text { Lisses, à paroi épaisse, } \\
\text { ellipsoïdales, amygdaliformes, } \\
\text { fortement colorées. } \\
1,3<\mathrm{Q}<1,7\end{array}$ \\
\hline Basides & $\begin{array}{l}\text { Taille: } 20 \times 6,6 \mu \mathrm{m} . \\
\text { Tétrasporiques (Figure } 4 \mathrm{I} \text { et L). }\end{array}$ & $\begin{array}{l}\text { Taille: } 22-37 \times 5-8 \mu \mathrm{m} . \\
\text { Tétrasporiques. }\end{array}$ \\
\hline Cheilocystides & $\begin{array}{l}\text { Taille: } 26,5 \times 7 \mu \mathrm{m} \text {. } \\
\text { Cylindriques, utriformes, } \\
\text { hyalines, lagéniformes ( Figure } 4 \mathrm{~F} \\
\text { et } \mathrm{G} \text { ). }\end{array}$ & $\begin{array}{l}\text { Taille: }(26) 32-52(76) \times 5-8 \\
\mu \mathrm{m} . \\
\text { Cylindriques, utriformes, } \\
\text { hyalines, lagéniformes. }\end{array}$ \\
\hline Hyphes des écailles & Largeur: $7-8 \mu \mathrm{m}$ & Largeur: $6-14 \mu \mathrm{m}$. \\
\hline Revêtement & $\begin{array}{l}\text { Absence d'une couche } \\
\text { gélatineuse (Figure 3E). }\end{array}$ & $\begin{array}{l}\text { Présence d'une couche } \\
\text { gélatineuse mince. }\end{array}$ \\
\hline
\end{tabular}


Tableau 3: Description macroscopique de Crepidotus mollis var. squamulosus.

\begin{tabular}{|c|c|c|}
\hline $\begin{array}{l}\text { Earactères } \\
\text { macroscopiques }\end{array}$ & $\begin{array}{l}\text { Crepidotus mollis var squamulosus. } \\
\text { Récolte de } 2009 \text { sur Eucalyptus } \\
\text { gomphocephala, Gharb (Maroc). }\end{array}$ & $\begin{array}{l}\text { Crepidotus calolepis var. } \\
\text { squamulosus Senn-Irlet } \\
\text { (1995) }\end{array}$ \\
\hline Chapeau & $\begin{array}{l}\text { Taille: } 40 \mathrm{~mm} . \\
\text { Forme: arrondie, circulaire, } \\
\text { flabelliforme, à marge largement } \\
\text { infléchie, villeux au point d'attache, } \\
\text { hygrophane; présence de méchules } \\
\text { rosâtres ou brunâtres (Figure 6A). } \\
\text { Couleur du fond jaune-brun clair. }\end{array}$ & $\begin{array}{l}\text { Taille: } 5-45 \mathrm{~mm} . \\
\text { Forme: arrondie, semi- } \\
\text { circulaire, spatulé à marge } \\
\text { largement infléchie, } \\
\text { hygrophane, villeux, } \\
\text { écailleux. } \\
\text { Couleur du fond [blanc] } \\
\text { sale ou jaune pâle. }\end{array}$ \\
\hline Stipe & $\begin{array}{l}\text { Visible chez les jeunes } \\
\text { fructifications. }\end{array}$ & $\begin{array}{l}\text { Visible chez les jeunes } \\
\text { fructifications. }\end{array}$ \\
\hline Lames & $\begin{array}{l}\text { Taille: } 12 \times 3 \mathrm{~mm} \text {. } \\
\text { Assez serrées, arquées, adnées } \\
\text { (Figure 6B). } \\
\text { Couleur: brunâtre. }\end{array}$ & $\begin{array}{l}\text { Taille: } 12-24 \times 1-3 \mathrm{~mm} \text {. } \\
\text { Serrées, étroites, arquées, } \\
\text { adnées. } \\
\text { Couleur: crème, } \\
\text { légèrement chamois. }\end{array}$ \\
\hline
\end{tabular}

Tableau 4: Description microscopique de Crepidotus mollis var. squamulosus

\begin{tabular}{|c|c|c|}
\hline $\begin{array}{l}\text { Espèces } \\
\text { Caractères } \\
\text { microscopiques }\end{array}$ & $\begin{array}{l}\text { Crepidotus mollis var. } \\
\text { squamulosus. } \\
\text { Récolte de } 2009 \text { sur Eucalyptus } \\
\text { gomphocephala, Gharb (Maroc). }\end{array}$ & $\begin{array}{l}\text { Crepidotus calolepis var. } \\
\text { squamulosus. } \\
\text { Senn-Irlet (1995) }\end{array}$ \\
\hline Spores & $\begin{array}{l}\text { Taille: } 7-10 \times 5-7 \mu \mathrm{m} . \\
\text { Lisses, à paroi épaisse, } \\
\text { ellipsoïdales, inéquilatérales, de } \\
\text { couleur rosâtre (Figure } 9 \mathrm{H} \text { ). } \\
1,3<\mathrm{Q}<1,7\end{array}$ & $\begin{array}{l}\text { Taille: } 8,5-12 \times 6-7,5 \mu \mathrm{m} . \\
\text { Lisses, à paroi épaisse, } \\
\text { ellipsoïdales, inéquilatérales à } \\
\text { amygdaliformes, fortement } \\
\text { colorées. } \\
1,3<\mathrm{Q}<1,7\end{array}$ \\
\hline Basides & $\begin{array}{l}\text { Taille: } 23 \times 8 \mu \mathrm{m} \text {. } \\
\text { Tétrasporiques (Figure } 9 \mathrm{G} \text { ). }\end{array}$ & $\begin{array}{l}\text { Taille: } 25-33 \times 6-8 \mu \mathrm{m} . \\
\text { Tétrasporiques. }\end{array}$ \\
\hline Cheilocystides & $\begin{array}{l}\text { Taille: } 29-32 \times 7-8 \mu \mathrm{m} \text {. } \\
\text { Cylindriques, flexueuses, hyalines. } \\
\text { Figure } 8(\mathrm{~F}) .\end{array}$ & $\begin{array}{l}\text { Taille: } 31-87 \times 4-40 \mu \mathrm{m} . \\
\text { Cylindriques, flexueuses, } \\
\text { hyalines, lagéniformes ou } \\
\text { septées. }\end{array}$ \\
\hline $\begin{array}{l}\text { Hyphes des écailles } \\
\text { Revêtement }\end{array}$ & $\begin{array}{l}\text { Largeur: } 8-10 \mu \mathrm{m} . \\
\text { Couche gélatineuse épaisse } \\
\text { (Figure 7D). }\end{array}$ & $\begin{array}{l}\text { Largeur: } 8-15(22) \mu \mathrm{m} . \\
\text { Couche gélatineuse épaisse. }\end{array}$ \\
\hline
\end{tabular}




\section{DISCUSSION}

Les observations de Malençon et Bertault (1975), montrent que Crepidotus mollis var. squamulosus est un grand Crepidotus gélatineux banal, fréquent au Maroc durant toute la saison des pluies, en particulier sur les troncs vivants de divers Eucalyptus sp.. Il présente un chapeau tantôt uni, tantôt moucheté de squames apprimées, brunâtres, plus ou moins espacées. Aussi, nous confirmons ces observations par nos récoltes du mois d'Octobre jusqu'à la fin du mois de Mars sur les troncs vivants d'Eucalyptus sp. dans la région du Gharb.

Or, Fries (1878) a décrit un Crepidotus calolepis auquel on serait d'autant plus tenté de rapporter la forme squameuse que plusieurs auteurs (Malençon et Bertault, 1975) y voient une simple variété du Crepidotus mollis. Cependant, selon Fries (1878), cité par Malençon et Bertault (1975), Crepidotus calolepis est un petit champignon, villeux, orné de fines squamules brunes, serrées et à chair non gélatineuse, ce qui ne s'accorde guère avec le Crepidotus mollis classique.

Alors que Senn-Irlet (1995) a signalé la présence d'une couche gélatineuse au niveau de l'épicutis de Crepidotus calolepis var. calolepis. Celui des spécimens récoltés dans le Gharb sur Eucalyptus sp. ne présente pas cette couche gélatineuse. Des centaines de coupes minces, transversales ou tangentielles, ne l'ont pas mis en évidence. La couche externe à hyphes couchées repose directement sur la couche à hyphes lâches (Figure 3C).

Les observations de Peric et Peric (2010) montrent que Crepidotus calolepis var. squamulosus se présente toujours avec un chapeau gélatineux, moucheté de squames brunâtres. Il pousse de manière solitaire ou grégaire, durant tout l'automne, ou en saison pluvieuse, sur troncs vivants et sur branches tombées d'Eucalyptus globulus.

Dans ce sens, Malençon et Bertault (1975), ont signalé l'existence de deux Crepidotus squamuleux, voisins mais différents. L'un, banal, de grande taille, franchement gélatineux et jaunâtre, simple forme pelucheuse de Crepidotus mollis, répondant à la variété squamulosus, l'autre petit, villeux, non ostensiblement gélifié, couvert de fines méchules serrées, qui serait le véritable Crepidotus calolepis de Fries. Mais, d'après ces deux auteurs, seul le premier existe au Maroc, et ce qu'ils ont signalé sous le nom de Crepidotus calolepis répond à la variété squamulosus, dont ils ont conservé désormais l'appellation de la forme pelucheuse ou méchuleuse de Crepidotus mollis.

Courtecuisse (2009) conserve le nom de Crepidotus mollis (Schaeff. ex Fr.) Kummer 1871 et var. squamulosus $\mathrm{P}$. Coutinho 1921, dans sa révision du genre Crepidotus (Flore des champignons supérieurs du Maroc tome II), dans le complément à la flore des champignons supérieurs du Maroc de Malençon et Bertault (1975). Il a signalé que Senn-Irlet (1995) adopte la présence de squamules comme critères distinctifs du taxon Crepidotus calolepis (au rang spécifique) que les chapeaux soient distinctement gélifiés ou non, contrairement à l'opinion développé par Malençon (1975), qui se base sur l'importance de la couche gélifiée pour rattacher ces dernières à Crepidotus mollis. Le maintien d'une variété (subtilement) macrospore et proposé par Senn-Irlet, qui adopte l'épithète squamulosus (comme Crepidotus calolepis var. squamulosus (Coutinho) Senn-Irlet (1995) retenue également par Malençon comme Crepidotus mollis var. squamulosus Coutinho (Courtecuisse, 2009).

Selon Roux (2006), la variété squamulosus Cout. possède des spores plus grandes (jusqu'à $12 \times 7,5 \mu \mathrm{m}$ ) et sa répartition se situe surtout dans la zone occidentale de la méditerranée (Espagne, Maroc, ....).

\section{Conclusion}

Crepidotus mollis var. squamulosus et Crepidotus calolepis, rencontrées dans la région du Gharb (Nord-Ouest du Maroc), fructifient ensemble sur les troncs vivants d'Eucalyptus sp. Les descriptions macroscopiques des deux espèces sont presque identiques, avec quelques différences 
au niveau de la taille et de la couleur du chapeau et des lames.

À part l'absence de la couche gélatineuse chez l'espèce Crepidotus calolepis, les descriptions que nous avons fournies pour les deux spécimens rencontrés dans la région du Gharb sont presque identiques à celles données par Senn-Irlet (1995).

\section{REFERENCES}

Boedyn K. 1966. Les Plantes du Monde. (Tome III): les Plantes sans Fleurs. Edition Hachette: Paris; 350 p.

Bouab RE. 2008. Composition avifaunistique et fonctionnement des rizières de la province de Sidi Kacem (Maroc). Bulletin de l'Institut Scientifique, Section Sciences de la Vie: Rabat, 30: 37-44.

Consiglio G, Setti L. 2008. Il Genere Crepidotus in Europa. Édition Associazione Micologica Bresadola: Trento; 344 p.

Courtecuisse R. 2009. Révision du genre Crepidotus. In Compléments à la Flore des Champignons Supérieurs du Maroc, de Malençon G, Bertault R (eds). Confédération Européenne Méditerranéenne: Nice; $775 \mathrm{p}$.

Loiseau J. 1951. Méthode Pratique pour la Recherche des Champignons sur le Terrain ( $3^{\text {ème }}$ édn). Vigot frères éditeurs: Paris; $211 \mathrm{p}$.

Malençon G, Bertault R. 1975. Flore des Champignons Supérieurs du Maroc (Tome II). Édition Faculté des Sciences de Rabat: Rabat; 540 p.

Maublanc A. 1926. Les Champignons de France (Tome II, $2^{\mathrm{e}}$ edn), Paul Lechevalier $237 \mathrm{p}$.

Norstein S. 1990. The genus Crepidotus (Basidiomycotina, Agaricales) in Norway. Fungiflora 2: Oslo; 115 p.

Peric B, Peric O. 2010. Crepidotus calolepis var. squamulosus un taxon inédit au Monténégro. Bulletin Mycologique et Botanique, 198: 11-19.

Roux P. 2006. Mille et un Champignons. Édition Roux: Sainte-Sigolène, France; $1223 \mathrm{p}$.

Senn-Irlet B. 1995. Le genre Crepidotus en Europe. Persoonia, 16: 1-80. 